Robotics 98: The 3rd Conference and Exposition/Demonstration on Robotics for Challenging Environments Sponsored by American Society of Civil Engineers April 26-30, 1998 Albuquerque, New Mexico

\title{
Flexible, Low-mass Robotic Arm Actuated by Electroactive Polymers and Operated Equivalently to Human Arm and Hand
}

\author{
Y. Bar-Cohen ${ }^{1}$, T. Xue ${ }^{1}$, M. Shahinpoor ${ }^{2}$, J. Simpson ${ }^{3}$, and J. Smith ${ }^{3}$
}

\begin{abstract}
Actuation devices are used for many space applications and there is increasing need to reduce their size, mass, cost and power consumption. To address this need, JPL is developing electroactive polymers (EAP) with emphasis on two EAP categories that induce large bending and longitudinal actuation strains. Comparison between EAP and the widely used transducing actuators shows that, while lagging in force delivering capability, these materials are superior in mass, power consumption and actuation strain levels. This study is concentrating on the development of effective EAPs and the enabling of mechanisms that employ their unique characteristics. Several EAP driven mechanisms that emulate human hand were developed including a gripper, manipulator arm and surface wiper. The manipulator arm is made of a composite rod that is lifted by a longitudinal rope actuator and has an end-effector gripper with bending EAP fingers allowing to grab and hold such objects as rocks. The EAP surface wiper operates like a human finger and can be used to remove dust from windows and solar cells. These EAP driven devices take advantage of the large displacement capability with less concern for their limited force actuation capability.
\end{abstract}

\section{INTRODUCTION}

Efficient miniature actuators that are light, compact and driven by low power are needed to support telerobotic requirements of future NASA missions. Generally, actuators are used to operate telerobotic devices that include robotic arms, rovers, etc. Other space applications include release mechanisms, antenna and instrument deployment, positioning devices, aperture opening and closing devices, and real-time compensation for thermal expansion in space structures, etc. Increasingly, there are

\footnotetext{
${ }^{1}$ Jet Propulsion Laboratory (JPL), Caltech., Pasadena, CA, yosi@jpl.nasa.gov

2 Artificial Muscles Research Institute, UNM, Albuquerque, NM

${ }^{3}$ Composites and Polymers Branch, NASA LaRC, Hampton, VA
} 
requirements to reduce the size, mass, and power consumption of actuation devices, and lower their cost. Electroceramics (piezoelectric and electrostrictive) offer effective, compact, actuation materials and they can be incorporated into ultrasonic motors, inchworms, translators and manipulators. In contrast to electroceramics, electroactive polymers (EAP) are emerging as new actuation materials [1] with displacement capabilities that cannot be matched by the striction-limited and rigid ceramics. Table 1 shows a comparison between the capability of EAP materials, electroactive ceramics (EAC) and shape memory alloys (SMA). As shown in Table 1, EAPs are lighter and their striction capability can be as high as two orders of magnitude more than EACs. Further, their response speed is significantly higher than SMAs. The authors' current study is directed towards taking advantage of these polymers' resilience and the ability to engineer their properties. The mass producibility of polymers and the fact that EAP do not require poling (in contrast to piezoelectric materials) help to produce them at low cost. EAPs can be easily formed in any various shapes and can be used to build micro-electro-mechanical systems (MEMS). They can be designed to emulate the operation of biological muscles [2-4] with unique characteristics of high toughness, large actuation strain constant and inherent vibration damping.

TABLE 1: Comparison of the properties of EAP, SMA and EAC

\begin{tabular}{|l|l|l|l|}
\hline \multicolumn{1}{|c|}{ Property } & \multicolumn{1}{|c|}{$\begin{array}{c}\text { Electroactive } \\
\text { polymers (EAP) }\end{array}$} & \multicolumn{1}{|c|}{$\begin{array}{c}\text { Shape memory alloys } \\
\text { (SMA) }\end{array}$} & $\begin{array}{c}\text { Electroactive } \\
\text { Ceramics (EAC) }\end{array}$ \\
\hline Actuation strain & $>10 \%$ & $<8 \%$ short fatigue life & $0.1-0.3 \%$ \\
\hline Force $(\mathrm{MPa})$ & $0.1-3$ & about 700 & $30-40$ \\
\hline Reaction speed & $\mu \mathrm{sec}$ to sec & sec to min & $\mu$ sec to sec \\
\hline Density & $1-2.5 \mathrm{~g} / \mathrm{cc}$ & $5-6 \mathrm{~g} / \mathrm{cc}$ & $6-8 \mathrm{~g} / \mathrm{cc}$ \\
\hline Drive voltage & $2-7 \mathrm{~V} / 10-100 \mathrm{~V} / \mu \mathrm{m}$ & NA & $50-800 \mathrm{~V}$ \\
\hline Consumed Power & m-watts & Watts & watts \\
\hline Fracture toughness & resilient, elastic & Elastic & fragile \\
\hline
\end{tabular}

The development of muscle actuators is involved with an interdisciplinary effort using expertise in materials science, chemistry, electronics, and robotics. At the initial phase of the authors' study efforts were made to identify electroactive polymers that offer large actuation strains. Two categories of EAPs were identified including (a) bending actuators - Perfluorinated Ion-exchange membrane platinum (IEMP) composites, and (b) longitudinal actuators - employing electrostatically activated EAPs. These two EAP actuators offer the capability to bend or stretch/extend, which essentially emulate the operation of biological muscles and limbs.

\section{IONOMERS AS BENDING EAP ACTUATORS}


The bending EAP actuator is composed of a perfluorinated ion exchange membrane, with chemically deposited platinum electrodes on its both sides. The formed muscle actuator is 0.18 -mm in thickness and formed in $1 \mathrm{x} 0.125$-inch strips weighing $0.1-\mathrm{g}$. To maintain the actuation capability of IEMP, it needs to be kept moist continuously. Efforts are currently being made to overcome this limitation and success was observed when using thick platinum electrodes where the voltage is limited to $<2-\mathrm{V}$ rather than the levels of 3-5 volts. Using such electrodes an IEMP film was demonstrated to actuate continuously for more than one million cycles. Another alternative that is considered is the use of encapsulation methods to form quasi-skin to protect the ionic constituents of IEMP composite films.

The structure and properties of the IEMP have been the subject of numerous investigations (see for example [5]). One of the interesting properties of this material is its ability to absorb large amounts of polar solvents, i.e. water. In order to chemically electrode IEMPs, platinum $(\mathrm{Pt})$ metal ions are dispersed throughout the hydrophilic regions of the polymer, and are subsequently reduced to the corresponding zero valent metal atoms. This results in the formation of a dendritic type electrode. When equilibrated with aqueous solutions these membranes are swollen and they contain a certain amount of water. Swelling equilibrium results from the balance between the elastic forces of the polymeric matrix and the water affinity to the fixed ion-exchanging sites and the moving counter ions. The water content depends on the hydrophilic properties of the ionic species inside the membrane and also on the electrolyte concentration of the external solution. To enhance the force actuation capability of IEMPs, techniques of producing thicker films as well as modification of the ionomer processing were investigated. Success was observed in processing the material to induce more than two times the strain with a higher response consistency. To better understand the actuation mechanism in ionomers the phenomena is studied and modeled. Also, alternative ionomer actuators are being searched.

Figure 1: A view of a surface wiper with a simulated window, where an ionomer is

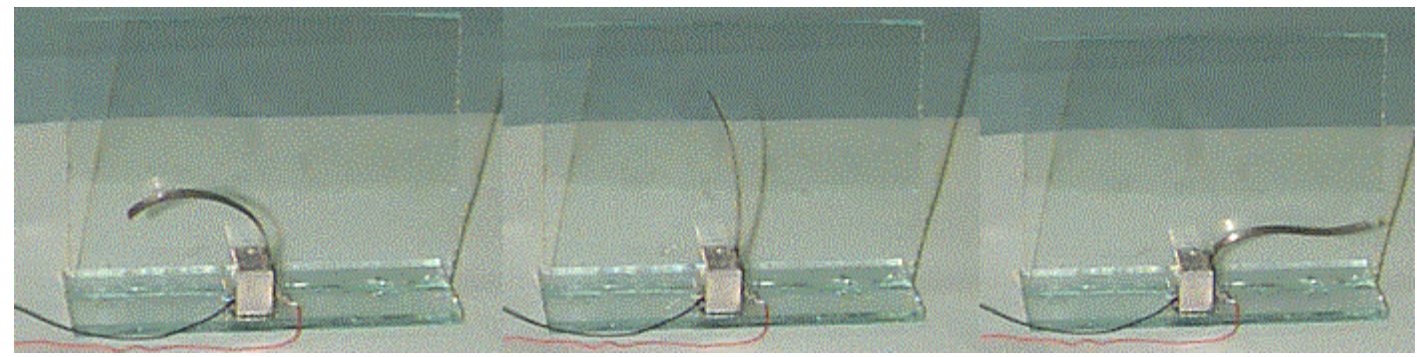

bending back and forth next to a glass plate (the slanted double image is a shadow).

When an external voltage is applied on an IEMP composite film, it bends towards the anode at a level that increases with the voltage (reaching saturation at about 6 or $7-\mathrm{V}$ ). Under AC voltage, the film undergoes swinging movement and the displacement level depends not only on the voltage magnitude but also on the frequency. Lower frequencies (down to 0.1 or $0.01 \mathrm{~Hz}$ ) lead to higher displacement (approaching 1 inch). The movement of the muscle is fully controllable by the applied electrical source 
but it is strongly affected by the water content that serves as an ion transport medium. The operation of the ionomer as a bending actuator is demonstrated in a configuration of a window surface wiper in Figure 1. As can be seen in this Figure, an ionomer film is attached to the surface of a glass plate and it is actuated left or right as desired by changing the polarity of the drive voltage. This ionomer film was driven by $2.5 \mathrm{~V}$ and 20-mW.

\section{LONGITUDINAL ELECTROSTATIC POLYMER ACTUATORS}

Polymers with low elastic stiffness and high dielectric constant can be used to induce large actuation strain by subjecting the material to an electrostatic field. These characteristics of polymers allow producing longitudinal actuators that operate similar to biological muscles. The governing principle is the response of the material to Coulomb forces between charged particles. Traditional electrostatic actuators are fabricated as a capacitor with parallel electrodes with a thin air gap between them. One of the major disadvantages of this type of actuators is their relatively low breakdown voltage. The authors adopted the approach that was reported in reference [4], where a longitudinal electrostatic actuator was made of dielectric elastomer film coated with carbon electrodes. The force (stress) that is exerted normally on such a film with compliant electrodes is as follows:

$$
P=\varepsilon \varepsilon_{0} E^{2}=\varepsilon \varepsilon_{0}(V / t)^{2}
$$

Where: $P$ is the normal stress, $\varepsilon_{0}$ is the permittivity of vacuum and $\varepsilon$ is the relative permittivity (dielectric constant) of the material, $E$ is the electric field across the thickness of the film, $V$ is the voltage applied across the film and $t$ is the thickness of the film.

Examining the equation above, it is easy to notice that the force magnitude is twice as large as that for the case of rigid parallel electrodes. To obtain the thickness strain the force needs to be divided by the elastic modulus of the film. Use of polymers with high dielectric constants and application of high electric fields allow inducing large forces and strains. To obtain the required electric field levels there is a need for either to use high voltage and/or employ thin films. For elastomers with low elastic modulus, it is reasonable to assume a Poisson's ratio of 0.5 . This means that the volume of the polymer is kept constant while the film is deformed under the applied field. As a result, the film is squeezed in the thickness direction causing expansion in the transverse plane. For a pair of electrodes with circular shape, the diameter and thickness changes can be determined using the following relation, where the second order components are neglected.

$$
\Delta D / D_{0}=(1 / 2) \Delta t / t_{0}
$$

Where: $D_{0}$ is the original diameter of the electrodes and $\Delta D$ is the resultant diameter change, $t_{0}$ is the original thickness and the $\Delta t$ is its change under electric activation.

To produce a longitudinal actuator with large actuation force, a stack of two silicone layers (Dow Corning Sylgard 186) was used with carbon electrodes on both sides of 
one of the layers. The two layers were rolled to form a rope as the one shown photographically next to a scale in Figure 2. The displacement in the rope cross section is a rotational one around the rope axis and it is constrained by interlaminar stresses. Therefore, the total actuation extension of the rope is proportional to its length and the resultant actuation force is proportional to the cross-section area normal to the axis. To develop an EAP muscle using such a rope, the length and diameter are used as design parameters, enabling the adaptation of the rope actuator to specific applications.

\section{ROBOTIC APPLICATIONS USING EAP ACTUATOR}

The availability of EAP actuators that can bend or extend/contract allows producing unique robotic devices that emulate human hands. The authors investigated several potential applications including gripper, robotic arm and surface wiper. As shown earlier, IEMP composite films are demonstrating a remarkable bending strain under a relatively low voltage drive, using a very low power. However, these ionomers are demonstrating a relatively low force actuation capability. Since IEMPs are made of a relatively strong material with a large strain capability, they were employed similar to the function of human fingers. In Figure 3, a gripper is shown using IEMP fingers in the form of an end-effector of miniature low mass robotic arms. The fingers move back and forth to allow opening similar to human hand, embracing the desired object and gripping on it. The hooks at the end of the fingers are function similar to fingernails to secure the gripped object.

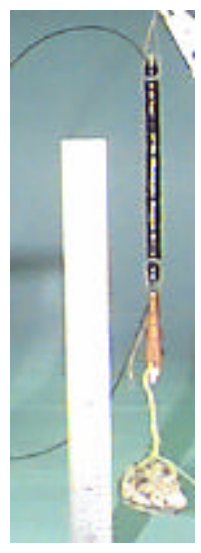

Figure 2: Electrostatic rope with a rock mounted on its bottom.

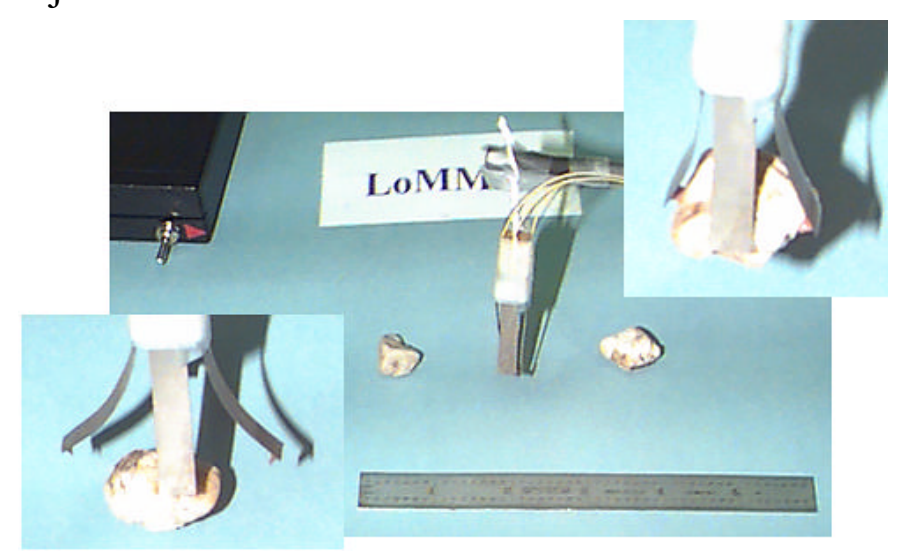

Figure 3: A 4-finger IEMP end-effector gripper lifting 10.3-g rock.

So far, multi-finger grippers that consist of 2- and 4-fingers were produced, where the 4-finger gripper lifted a mass of 10.3-g. This gripper prototype was mounted on a 5$\mathrm{mm}$ diameter graphite/epoxy rod to form lightweight arm. The gripper was driven by 2 to $5-\mathrm{V}$ square wave signal at a frequency of $0.1-\mathrm{Hz}$ to allow sufficient time to perform a demonstration of the gripper capability. To operate the gripper its fingers are opened and the gripper is brought near the object to be collected. At this point the fingers are closed and the object is lifted. The demonstration of the gripper capability to lift a rock was intended to pave the way for a future application to planetary sample 
collection tasks providing miniature ultra-dexterous and versatile tool. To allow lifting the robotic arm, a set of two ropes was used as shown in Figure 4. One rope actuates the arm by tilting its balance and its lifting displacement is determined by the ratio between its connection distance from the pivot point compared to the gripper distance. The other rope is a longer one and is connected directly to the gripper that is lifted or dropped as a function of the rope actuation displacement. Figure 4 shows the full robotic arm with the two longitudinal rope actuators and the gripper with ionomer fingers gripper.

Lesson learned from Viking and Mars Pathfinder missions indicates that the operation on Mars is involved with an environment that causes the accumulation of dust on the hardware surfaces. The dust accumulation is a critical problem that hampers long-term operation of optical instruments and degrades the produced power efficiency of solar cells. To remove dust from surfaces one can use a similar mechanism as the windshield wipers of cars. Unfortunately, conventional surface wiping mechanisms are cumbersome, heavy, power gazzler and cannot be practical for such tasks as cleaning individual solar cells. On the other hand, IEMP bending actuator has the ideal characteristics of surface wiper. As shown in Figure 1, a simple, small, lightweight, low power consuming surface wiper can be constructed using an ionomer film. The ionomer responds to activation signals at the millisecond range and the angle of bending can exceed 180 degrees span and can cover 1.0 - 1.5 inch diameter of a circular area using about 1.5 - 2.0 inch long wiper. The wiper element can be set straight in the middle of the desired area and activated to sweep left and right by switching the electric field polarity. Also, it can be set on the side of the desired area and activated in one direction.

FIGURE 4: A view of the robotic arm that is driven by EAP actuators.

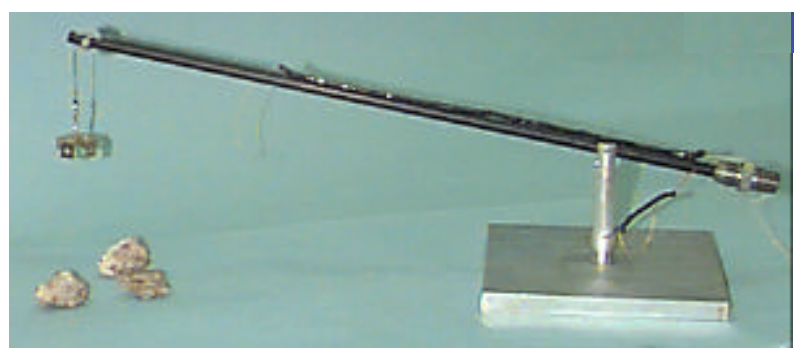

\section{CONCLUSIONS}

Two types of electroactive polymer actuators, which induce large displacement actuation, were employed in this study to develop robotic devices that emulated human hands. While the material performance is being enhanced, methods of controlling the actuation performance are being investigated. IEMPs are offering a large bending actuation and allow emulating the dexterity of human hand using lightweight material that consumes low power and is inexpensive to produce. For longitudinal displacement actuation, electrostatically activated films were rolled to form ropes and to serve equivalently to biological muscles. These electroactive polymers are showing a superior actuation displacement, mass, cost, power consumption and fatigue 
characteristics over conventional electromagnetic, EACs and SMAs. While the force actuation capability of EAPs is limited, their actuation displacement levels are unmatched. Telerobotic devices were constructed using EAP and allowed actuation of unique mechanisms. A multi-finger gripper was demonstrated to have large finger opening and closing with great mass carrying capability. A robotic arm was constructed similar to human hand using a composite rod, electrostatically driven rope and a 4-finger IEMP gripper as end-effectors. Currently, the practical application of IEMPs is constrained by the need to maintain the ionic constituents and preventing the film from drying. The equivalent of a biological skin is being investigated to protect the ion content of IEMP films. Encapsulation techniques are being investigated to assure the moisture containment and so far success was observed when using thicker platinum electrodes and voltage levels below 2-volts. To address the issue of dust on Mars, a unique surface wiper that is equivalent to a moving human finger was developed to allow removal of dust from windows and solar cells using low power, light-weight ionomer films.

\section{ACKNOWLEDGMENT}

The results reported in this manuscript were obtained under a TRIWG task, Code S. Mr. David Lavery and Dr. Chuck Weisbin are the Managers of TRIWG. The authors would like also to express their appreciation of Brian Lucky, Cinkiat Abidin, Marlene Turner and Harry Mashhoudy, who are students at the Integrated Manufacturing Engineering Dept. of UCLA. These students contributed greatly to the fabrication of the robotic arm and the electrostatic rope muscle.

\section{REFERENCES}

1. Furukawa and J. X. Wen, "Electrostriction and Piezoelectricity in Ferroelectric Polymers," Japanese Journal of Applied Physics, Vol. 23, No. 9, pp. 677-679, 1984.

2. I. W. Hunter and S. Lafontaine, "A comparison of muscle with artificial actuators," IEEE Solid-State Sensor and Actuator Workshop, pp. 178-165, 1992.

3. M. Shahinpoor, "Continuum electromechanics of ionic polymeric gels as artificial muscles for robotic applications," Smart Materials and Structures, Vol. 3, pp. 367372, 1994.

4. R. Kornbluh, R. Pelrine and J. Joseph, "Elastomeric dielectric artificial muscle actuators for small robots," Proceeding of the $3^{\text {rd }}$ IASTED International Conference, Concun, Mexico, June, 14-16, 1995.

5. C. Heitner-Wirguin, "Recent advances in perfluorinated ionomer membranes: Structure, properties and applications," Journal of Membrane Science, V 120, No. 1, pp. 1-33, 1996. 